\title{
Optical electrocorticogram (OECoG) using wide-field calcium imaging reveals the divergence of neuronal and glial activity during acute rodent seizures
}

Andy G. S. Daniel, Philippe Laffont, Mingrui Zhao,Hongtao Ma, and Theodore H. Schwartz

Departments of Neurosurgery and Neuroscience, Weill Cornell Medical College, New York, NY, USA

Key Words: Astrocyte, Calcium, Epilepsy, Glia, Ictal, Imaging, Interictal, Rat, Seizure, 4-Aminopyridine

To whom correspondence may be addressed:

Hongtao Ma, Ph.D.

Departmentof Neurological Surgery, Weill Medical College of Cornell University, 525 East 68th Street, Box 99, New York, NY 10065

Phone: 212-746-5516

Fax: 212-746-7961

E-mail: hom2001@med.cornell.edu 


\begin{abstract}
The role of glia in epilepsy has been widely debated. Using in vivo bulk loading of calcium dyes we imaged neuronal and glial activity in an acute pharmacologic rodent model of neocortical seizures. Optical calcium-based ECoG maps revealed that neuronal waves propagated rapidly and remained mostly confined to the seizure focus. Glial waves were triggered by ictal onset but propagatedslowly in a stereotypical fashion far beyond the seizure focus. Although related at their onset, the divergence of these two phenomena during seizure evolution calls into question their interdependence and the criticality of the role of glia in seizure onset and neurovascular coupling.
\end{abstract}




\section{Introduction}

Over the last few decades, the role of glial cells in the brain has been expanded from merely providing structural support and nourishment to actively maintaining and modulating neuronal function[1-3]. Glia sense and respond to synaptic activity through activation of different neurotransmitter receptors and transporters[4]. Furthermore, a crucial role for gliahas been postulated in the neurovascular coupling process[5, 6]. In addition, glia have been hypothesized to play an important role in epilepsy[7, 8]. Glia modulateneurotransmitters such as glutamate and adenosine, which can promote neuronal synchrony, enhance neuronal excitability, regulate inhibition and redistribute elevated potassium[4]. Glia can also mediate spreading calcium waves through gap junctions[9].However, the role of glia in epileptogenesis and neurovascular coupling is controversial and studies have also shown contradictory evidence against the significance of glia as causative in these roles[10, 11].

Wide-field calcium imaging can be used to visualize calcium dynamics in the cortex as a means of high resolution mapping of neuronal and glial activity with very high temporal resolution and wide spatial sampling[12-14]. These images provide a type of optical electrocorticography (OECoG). Prior in vitro studies during epileptiform events have shown that glial activity occurs in 
stereotypical slow waves that occur spontaneously and are unrelated to neuronal activity and not linked to seizure onset[15]. We used wide field in vivo calcium imaging in a pharmacologic model of acute epilepsy to investigate the temporal and spatial relationship between neuronal and glial activity as a means to determine their spatial and temporal overlap as a measure of interdependence.

\section{Materials and Methods}

\section{Animal Preparation}

All experimental procedures were approved by the Weill Cornell Medical College Animal Care and Use Committee following National Institutes of Health guidelines. Adult male Sprague-Dawley rats (200-350 g) were anesthetized with isoflurane in $70 \% \mathrm{~N}_{2}: 30 \% \mathrm{O}_{2}, 4 \%$ induction, and 1.5-2\% maintenance. Body temperature was maintained at $37^{\circ} \mathrm{C}$ with a regulated heating blanket (Harvard Apparatus, Holliston, MA). The heart rate, $\mathrm{pO}_{2}$, and the end tail carbon dioxide $\left(\mathrm{ETCO}_{2}\right)$ were carefully monitored with a small animal Capnograph (Surgivet, Waukesha, WI) and were maintained stable throughout the experiment (heart rate: 250-300 pulse/minute, pO2 > 90\%, ETCO2 25-28 mmHg). The head was fixed in a stereotaxic frame.

\section{Dye Staining}


Convection enhanced delivery (CED) wasused to bulk loadthe entire neocortex with the calcium indicator dye Oregon Green 488 BAPTA-1 AM (OGB1, Life Technologies, Grand Island, New York)[14]. This technique involves a long continuous injection of calcium dyes rather than the more common approach using multiple small injections[16]. A 5x8 mm craniotomy window was opened overthe stained hemisphere between lambda and bregma, and the exposed brain was covered with silicon oil (12,500 centistoke) to preserve cortical moisture.

Epileptiform Model and Electrophysiology

4-Aminopyridine (4-AP, Sigma-Aldrich, $15 \mathrm{mM}, 0.5 \mu \mathrm{L}$ ) was injected 300$500 \mu \mathrm{m}$ into the cortex to generate epileptiform events, through a glass microelectrode using a Nanoject II injector (Drummond Scientific, Broomall, PA) which also enabled the recording of the local field potential (LFP)[17]. Utilizing a

DAB-S system, the LFP was amplified and band-pass filtered (1-500 Hz). A CED Power 1401 then digitized the signal at 1000. The LFP data were recorded via the Spike2 software (Cambridge Electronic Design, Cambridge, UK).

Optical Recording

For wide-field calcium imaging, a CCD camera (Dalsa camera in Imager 3001, Optical Imaging, Rehovot, Israel) using a tandem lens $(50 \times 50 \mathrm{~mm})$ 
arrangement was focused 300-400 $\mu \mathrm{m}$ below the cortical surface. A $470 \pm 10-\mathrm{nm}$ light-emitting diode (LED) was employed as the illumination source for OGB-1 with a 510-nm long-pass being placed before the camera (Fig. 1). The illumination was guided to the cortex with an optical fiber. The temporal resolution was $110 \mathrm{~Hz}$ with a pixel resolution of $\sim 50 \mu \mathrm{m} \times 50 \mu \mathrm{m}$.

\section{Data Analysis}

Custom written MATLAB programs were used to separate the OGB-1 signals using first order 1-Hz high-pass and low-pass Butterworth filters as well as to perform additional analyses. To increase the signal-to-noise ratio, spatial lowpass filtering was achieved by convolution with a Gaussian kernel ( $\sigma=3$ pixels).

Glial activity was separated from neuronal activity using frequency filtering. The high frequency component (HF, >1 Hz), which showed a similar waveform as the LFP, previously called an optical electrocorticogram (OECoG), mainly reflected the neuronal activity of the neuropil (pre-synaptic axonal activity) and action potentials from the somata[18]. The low frequency component (LF, <1 Hz) mainly reflected the activity of glia[12-14].

The spatial extent of the glial wave was determined by thresholding the data at $25 \%$ of the maximal amplitude based on the signal-to-noise ratio of our actual 
data [19-21].Correlation coefficient (CC) maps were employed to display the spatial propagation of neuronal activity. For spatial propagation of ictal events, we calculated the CC between the LFP and HF from each individual pixel or between the HF trace from the 4-AP injection site with each pixel.

\section{Results}

A few minutes after the local injection of 4-AP, ictal events could be recorded from the 4-AP/LFP electrodeand calcium imaging of the OECoG[17].These events began with a heralding spike followed by low voltage high frequency activity that evolved into periodic spike-and-wave events[22]. Interictal spikes also occurred between seizures (Fig. 2).

Neuronal versus glial activity in the focus and surround

Frequency filtering revealed a clear difference between HF (neuronal) and LF (glial) activity. In the seizure focus, HF maps successfully revealed the location of the calcium events underlying both small amplitude interictal spike as well full blown seizuresas evidenced by the similarity to the adjacent LFP recording. These results confirm the sensitivity of our CED bulk loading technique for wide-field calcium imaging of the neuropil (Fig. 2). The glial activity, on the other hand consisted of a slowly evolving wave of activity. Each glial wave was clearly 
initiated by the onset of the seizure and did not occur independently of the seizure. In certain instances, the calcium signal showed that the interictal spikes were clearly arising from the seizure focus based on the amplitude and width. In other instances the interictal spikes were higher in amplitude and narrower in width in the surround and likely arose from the surround and not the focus, possibly a form of network inhibition (Fig. 2). With regards to the neuronal activity in the surround,although the herald spike propagated to the surround, the seizure remained focal as evidenced by the absence of calcium activity in the surround at the onset in the evolution of the seizure. As the seizure evolved, spike and wave activity developed in thesurround, which was independent from the ongoing seizure activity in the focus. The glial activity in this distant region of cortex was less well formed and became evident later in the seizure, indicating delayed recruitment (Fig. 2).

Spatial propagation of neuronal and glial activity during ictal events

With wide field imaging we could also evaluate the spatial spread of the neuronal and glial activity. Neuronal activity consisted of rapidly propagating waves whose spatial extent remained relatively stable and focal with occasional waves that propagated to the surround. The glial wave, on the other hand, started in the focus and slowly marched to the surround, far beyond the neuronal activity, asa 
stereotypical all-or-none event. The average area of the glialactivity (16.3 \pm 3.6

$\mathrm{mm}^{2}$ )was much larger than the neuronal activity $\left(11.0 \pm 4.1 \mathrm{~mm}^{2}, \mathrm{n}=29\right.$ seizures; $\mathrm{n}=10$ rats; $\mathrm{P}<0.001$, paired t-test) (Fig. 3). Seizure duration also differed between the two signals with neuronal activity (34.7 \pm 4.9 s) outlasting the activation of the glial wave (23.7 $\pm 4.7 \mathrm{~s}, \mathrm{n}=8$ seizures; $\mathrm{n}=4$ rats; $\mathrm{P}<0.001$, paired t-test $)$.

\section{Discussion}

We used bulk loading of calcium dye and wide field imaging to create optical ECoG maps of neuronal and glial activity during acute in vivo

pharmacologic ictal and interictal events. These OECoG maps are sensitive enough to localize the onsets of individual interictal spikes and ictal onset zones as well as the propagation of each individual epileptic wave as the seizure evolves. Contrary to prior in vitro studies[15], we find that a slowly propagating calcium wave that spreads throughout the glial syncytium is triggered simultaneously with ictal events. However, this ictal glial wave spreads well beyond the high frequency activity that occurs in the neurons and their axons that make up the neuropil of the epileptic focus and appears spatiotemporally uncoupled from neuronal activity.

In vivo bulk calcium imaging has been used to map sensory events in normal cortex generally with multiple small injections of calcium dyes[16]. We developed a technique for loading the entire cortex homogeneously, which is particularly 
useful in mapping epileptic events[14]. Prior studies using two-photon imaging of individual neuronal and glial cell bodies have demonstrated that neuronal and glial events can be distinguished based on the rise and decay of the signal, being rapid in neurons and slow in glia[12, 13]. The neuronal signal appears to arise primarily from subthreshold pre-synaptic activity in axons as well as cell bodies firing action potentials[18]. We separated the neuropil and gliapil compartments using these well-defined and established frequency criteria.

\section{Calcium imaging of interictal and ictal neuronal activity}

Although these results are somewhat preliminary, several interesting observations can be made from these wide field imaging experiments. With respect to the neuropil, there appears to be a dynamic interplay between the focus and surrounding cortex during interictal and ictal events. With regards to interictal events, the OECoG calcium maps reveal that interictal events may begin outside of the ictal onset zone, even though they may appear to be arising from within the onset zone on limited electrical recordings. This is one of the limitations of electrical recordings, which is sampling. OECoG allows simultaneous sampling of large areas of cortex and reveals that interictal spikes may arise from outside of the seizure onset zone and propagate into the focus. We can only hypothesize that these distant spikes may play an inhibitory role; an attempt by the extended 
neuronal network to prevent the onset of an impending ictal event.Ictal events, on the other hand, consist of rapidly propagating waves of activity, as can also be seen using voltage sensitive dyes[23]. These events do not propagate throughout the cortex but remain restricted to the focus and, as opposed to a Jacksonian march model, continuously recruit a constant area of cortex from the start of the seizure. In the surrounding cortex, the OECoG reveals independent epileptiform events that are clearly temporally triggered by the evolution of the seizure but remain desynchronized. Perhaps these distant interictal events indicate paroxysms of network inhibition that herald the impending termination of the seizure.

\section{Calcium imaging of glial activity}

The glial signal appears to be a stereotypical wave that is initiated in the seizure onset zone and triggered by seizure onset. Calcium waves such as these have been described in normal cortex and appear to spread through gap junctions[9]. However, we show that these reactive waves arenot independent from the seizure but rather initiated by it. These glial waves should not be confused with spreading depression, which is a neuronal event that spreads at 3-5 $\mathrm{mm} / \mathrm{min}[24,25]$. These glial epileptic waves spread more rapidly and do not cause hyperpolarization of the cortex. Spatially, although these waves begin in the focus, they do not appear to overlap with the neuronal activity in the focus either in space 
or time once they are triggered. This independence and divergent evolution of the epileptic glial wave calls into question the allegedly crucial role of glia in epileptogenesisand neurovascular coupling, which has recently been questioned in several in vitro studies[7, 8, 10].

\section{Conclusions}

In this study, we have demonstrated that the rapid neuronal and slow glial waves associated with seizures, although correlated at their onset, evolve in unique divergent patterns. Calcium imaging provides an ideal optical method for high temporal resolution, wide spatial sampling to study these two divergent phenomenon.

\section{Acknowledgments}

This project was supported by the National Science Foundation, NSF-1264948 (H. M. and T. H. S.) and Citizens United for Research in Epilepsy Foundation (H. M.). 


\section{Disclosure}

The authors have no conflicts of interest to disclose.

\section{References}

[1] Araque A, Parpura V, Sanzgiri RP, Haydon PG. Tripartite synapses: glia, the unacknowledged partner. Trends Neurosci 1999;22: 208-15.

[2] Sasaki T, Kuga N, Namiki S, Matsuki N, Ikegaya Y. Locally synchronized astrocytes. Cereb Cortex 2011;21: 1889-900.

[3] Nedergaard M, Ransom B, Goldman SA. New roles for astrocytes: redefining the functional architecture of the brain. Trends Neurosci 2003;26: 52330 .

[4] Haydon PG, Carmignoto G. Astrocyte control of synaptic transmission and neurovascular coupling. Physiol Rev 2006;86: 1009-31.

[5] Ghosh A, Wyss MT, Weber B. Somatotopic astrocytic activity in the somatosensory cortex. Glia 2013;61: 601-10.

[6] Otsu Y, Couchman K, Lyons DG, Collot M, Agarwal A, Mallet JM, Pfrieger FW, Bergles DE, Charpak S. Calcium dynamics in astrocyte processes during neurovascular coupling. Nat Neurosci 2015;18: 210-8.

[7] Tian GF, Azmi H, Takano T, Xu Q, Peng W, Lin J, Oberheim N, Lou N, Wang X, Zielke HR, Kang J, Nedergaard M. An astrocytic basis of epilepsy. Nat Med 2005;11: 973-81.

[8] Gomez-Gonzalo M, Losi G, Chiavegato A, Zonta M, Cammarota M, Brondi M, Vetri F, Uva L, Pozzan T, de Curtis M, Ratto GM, Carmignoto G. An excitatory loop with astrocytes contributes to drive neurons to seizure threshold. PLoS Biol 2010;8: e1000352.

[9] Cornell-Bell AH, Finkbeiner SM, Cooper MS, Smith SJ. Glutamate induces calcium waves in cultured astrocytes: long-range glial signaling. Science 1990;247: 470-3. 
[10] Fellin T, Gomez-Gonzalo M, Gobbo S, Carmignoto G, Haydon PG. Astrocytic glutamate is not necessary for the generation of epileptiform neuronal activity in hippocampal slices. J Neurosci 2006;26: 9312-22.

[11] Bonder DE, McCarthy KD. Astrocytic Gq-GPCR-linked IP3R-dependent $\mathrm{Ca} 2+$ signaling does not mediate neurovascular coupling in mouse visual cortex in vivo. J Neurosci 2014;34: 13139-50.

[12] Schulz K, Sydekum E, Krueppel R, Engelbrecht CJ, Schlegel F, Schroter A, Rudin M, Helmchen F. Simultaneous BOLD fMRI and fiber-optic calcium recording in rat neocortex. Nat Methods 2012;9: 597-602.

[13] Gobel W, Helmchen F. In vivo calcium imaging of neural network function. Physiology (Bethesda) 2007;22: 358-65.

[14] Ma H, Harris S, Rahmani R, Lacefield CO, Zhao M, Daniel AG, Zhou Z, Bruno RM, Berwick J, Schwartz TH. Wide-field neocortical calcium dye imaging using a convection-enhanced loading technique combined with simultaneous multiwavelength imaging of voltage-sensitive dyes and hemodynamic signals. Neurophotonics 2014;1: 015003.

[15] Tashiro A, Goldberg J, Yuste R. Calcium oscillations in neocortical astrocytes under epileptiform conditions. J Neurobiol 2002;50: 45-55.

[16] Stosiek C, Garaschuk O, Holthoff K, Konnerth A. In vivo two-photon calcium imaging of neuronal networks. Proc Natl Acad Sci U S A 2003;100: 731924.

[17] Schwartz TH, Bonhoeffer T. In vivo optical mapping of epileptic foci and surround inhibition in ferret cerebral cortex. Nat Med 2001;7: 1063-7.

[18] Kerr JN, Greenberg D, Helmchen F. Imaging input and output of neocortical networks in vivo. Proc Natl Acad Sci U S A 2005;102: 14063-8.

[19] Chen-Bee CH, Kwon MC, Masino SA, Frostig RD. Areal extent quantification of functional representations using intrinsic signal optical imaging. $\mathrm{J}$ Neurosci Methods 1996;68: 27-37.

[20] Berwick J, Johnston D, Jones M, Martindale J, Martin C, Kennerley AJ, Redgrave P, Mayhew JE. Fine detail of neurovascular coupling revealed by 
spatiotemporal analysis of the hemodynamic response to single whisker stimulation in rat barrel cortex. J Neurophysiol 2008;99: 787-98.

[21] Ma H, Zhao M, Suh M, Schwartz TH. Hemodynamic surrogates for excitatory membrane potential change during interictal epileptiform events in rat neocortex. J Neurophysiol 2009;101: 2550-62.

[22] Zhao M, Ma H, Suh M, Schwartz TH. Spatiotemporal dynamics of perfusion and oximetry during ictal discharges in the rat neocortex. J Neurosci 2009;29: 2814-23.

[23] Ma H, Zhao M, Schwartz TH. Dynamic neurovascular coupling and uncoupling during ictal onset, propagation, and termination revealed by simultaneous in vivo optical imaging of neural activity and local blood volume. Cereb Cortex 2013;23: 885-99.

[24] Leo AAP. Spreading depression of activity in the cerebral cortex. J Neurophysiol 1944;7: 359-90.

[25] Ba AM, Guiou M, Pouratian N, Muthialu A, Rex DE, Cannestra AF, Chen JW, Toga AW. Multiwavelength optical intrinsic signal imaging of cortical spreading depression. J Neurophysiol 2002;88: 2726-35. 


\section{Figure Legends}

Figure 1. Experimental setup. Diagram for OGB-1 Calcium (Ca) imaging. The Ca illumination is filtered with a $470 \mathrm{~nm}$ filter and directed to the cortex via two fiber optic cables. The resulting fluorescence is then filtered with a $510 \mathrm{~nm}$ long pass filter placed in front of the camera.

Figure 2. Interictal spike and ictal event shown in local field potential (LFP) and calcium signal, the latter separated into its high frequency (HF) and low frequency

(LF) components in the ictal focus (blue) and surrounding distant (red) cortex. The top left shows the field of view and location of the 4-AP and LFP electrodes. When separated into its frequency components, the HF trace at the focus (blue) shows an optical electrocorticogram (OECoG) that closely resembled the LFP. In the focus, an interictal spike appears prior to the onsets of a seizure, characterized by a large heralding spike followed by the onset of low voltage high frequency activity. The LF trace shows a slowly propagating wave of glial activity. The onset of this activity correlates with the onset of the ictal event. In the surrounding cortex further away (red) the interictal spike appears to be higher in amplitude and narrower and thus arising from the surround. During the ictal event there is a brief interictal event and then a series of spike and wave events rather than an evolving seizure. The LF trace shows delayed onset of the glial wave. 
Figure 3. The spatial distribution of HF (neuronal) and LF (glial) activity during a seizure. (A) The top left shows the field of view and location of the 4-AP and LFP electrodes. The blue box indicates the area from which the Ca trace and subsequent HF and LF are derived. (B) Spatial propagation of HF and LF activity throughout ictal event. The seizure spread is shown at $6 \mathrm{~s}$ intervals. (I) Glial propagation during seizure. The glial activity begins at the seizure focus and propagates to the rest of the hemisphere as a slowly propagating wave with onset in the focus and offset in the surround. The wave has spread several $\mathrm{mm}$ in only 6 seconds and reaches its maximum around $18 \mathrm{~s}$. (II) The neuronal activity also begins at the seizure focus but the activity, although consisting of rapidly propagating waves, does not progressively march across the cortex. Rather the activity remains conserved to a relatively focal area the majority of which is involved very early in seizure evolution. Occasionally some waves propagate further laterally (as seen at 36s) but the majority of the activity stays within the focus (III) Spatial overlap of glial and neuronal activity during seizure evolution. The glial waves clearly propagate further into the surrounding areas as a slowly moving wave that starts in the focus and spreads laterally whereas the neuronal activity stays within the focus. 


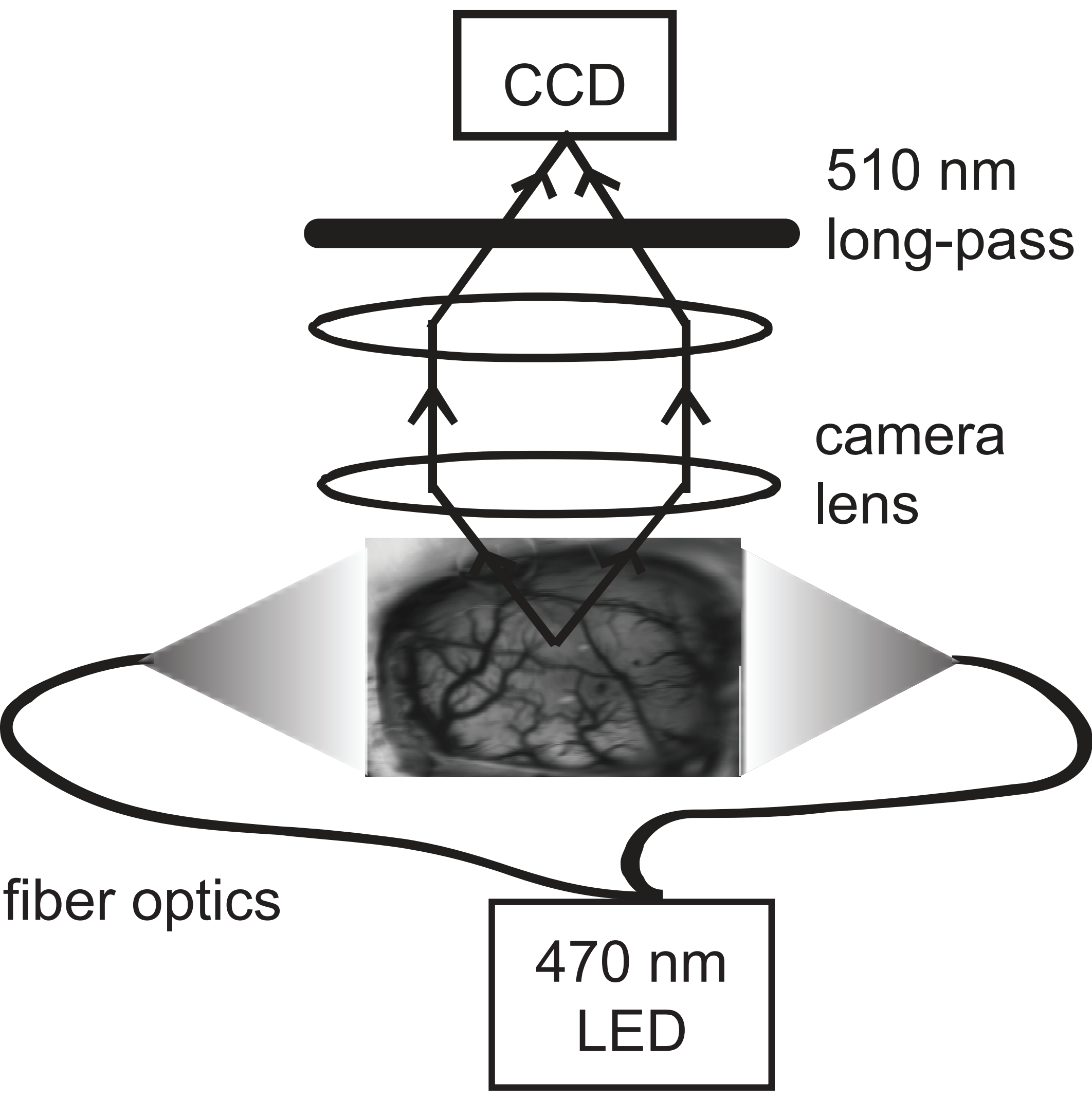

fiber optics

$470 \mathrm{~nm}$
LED

$510 \mathrm{~nm}$

long-pass

camera

lens

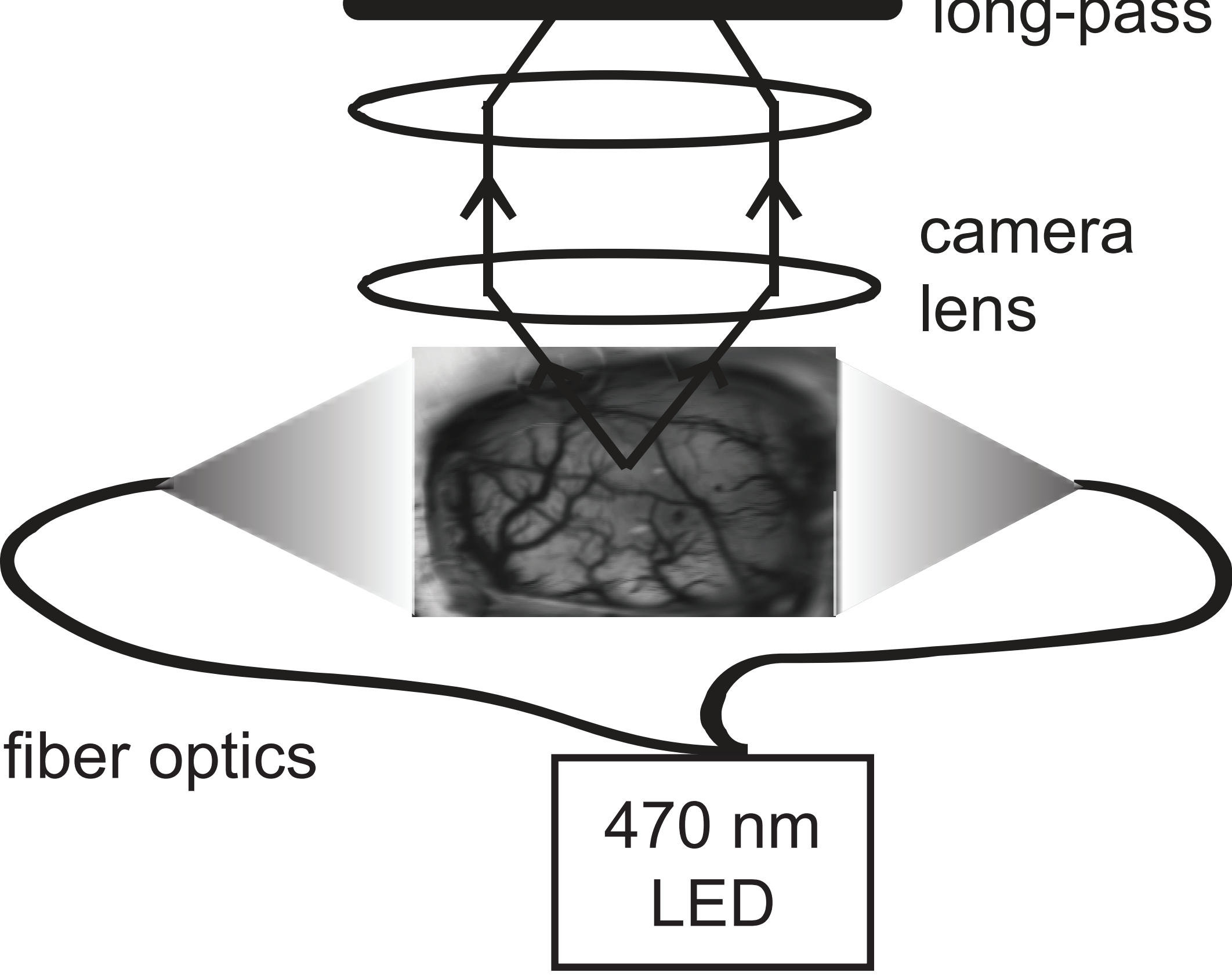

Figure 1

\section{Figure 1}



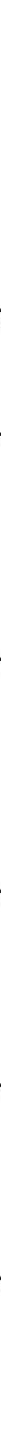

Figure 2 
A

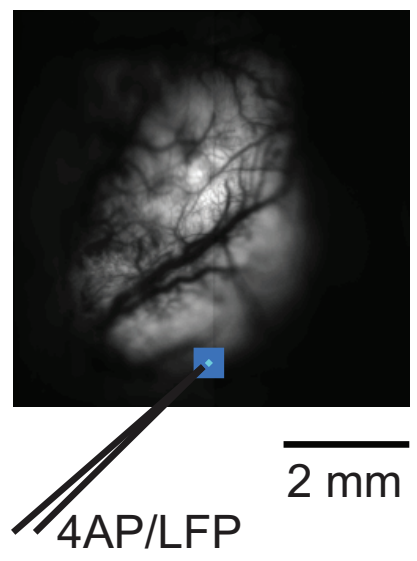

LFP

$\mathrm{Ca}$

HF Maty LF
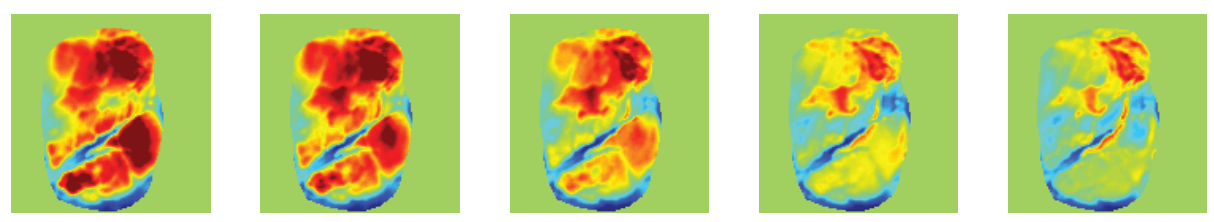

$5 \%$ $\mathrm{dF} / \mathrm{F}$ $-5 \%$
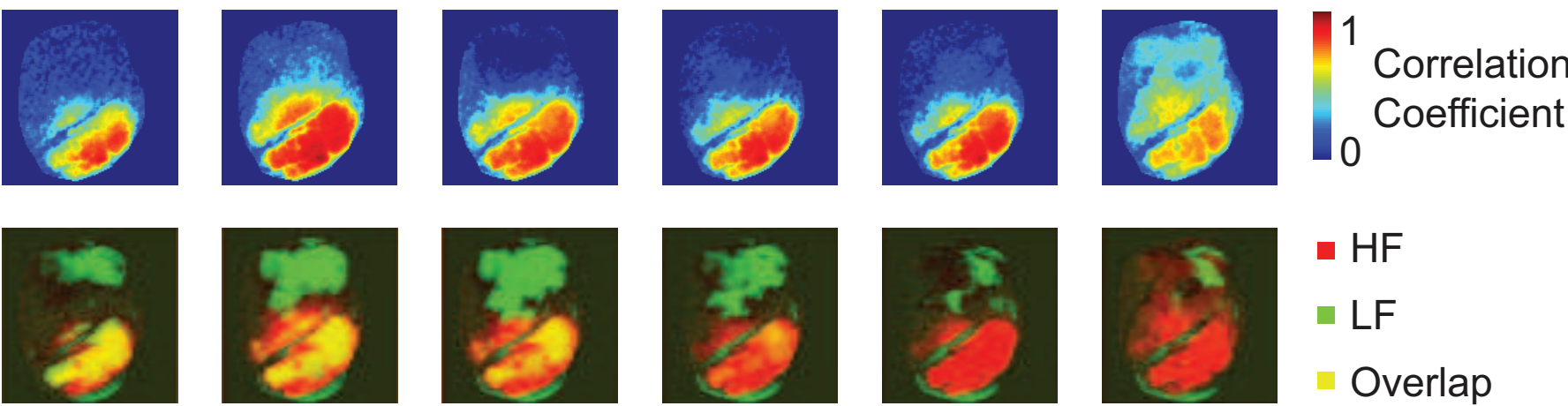

6

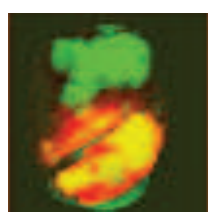

12

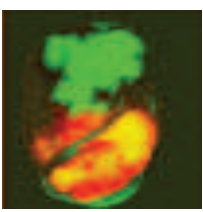

18

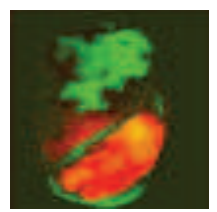

24

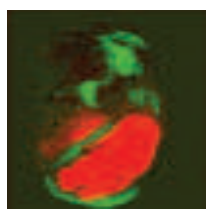

30

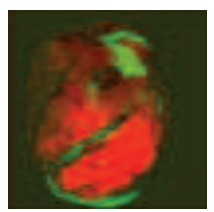

$36 \mathrm{~s}$
- HF

- LF

- Overlap

Figure 3 